\title{
Gender Differences in the Microanatomy of Skin of Sole in Humans
}

\section{IJCRR \\ Section: Healthcare \\ ISI Impact Factor \\ (2019-20): 1.628 \\ IC Value (2019): 90.81 \\ $\operatorname{SJIF}(2020)=7.893$}

(c) (i) (3)

Copyright@IJCRR

\section{Pankaj Arvind Kumar ${ }^{1}$, Dande Kaweri2*, Rani Anita ${ }^{3}$, Chopra Jyoti ${ }^{3}$, Rani Archana ${ }^{3}$, Srivastav Saurabh ${ }^{4}$}

'Professor Junior Grade, Department of Anatomy, King George's Medical University, UP, Lucknow, India, ${ }^{2}$ Post Graduate Scholar, Department of Anatomy, King George's Medical University, UP, Lucknow, India; ${ }^{3 P}$ Professor, Department of Anatomy, King George's Medical University, UP, Lucknow, India; 4 Senior Resident, Department of Pediatrics Surgery, King George’s Medical University, UP, Lucknow, lndia.

\section{ABSTRACT}

Introduction: It is known that actual values of thickness of epidermis of the skin and its variations with body site, age and sex are important in fields of medical and biological research. The variations in skin reaction to certain stimuli could be due to biological factors such as epidermal thickness, dermal thickness, distribution of epidermal appendages etc.

Aims: Aim of the present study was to establish variations in the number of different components of skin in human cadavers.

Material and Methods: Skin was procured from the sole of six freshly formalin-fixed human cadavers. Out of these three were males and three were females. The age of male and female cadavers ranged between 60 to $70 y e a r s$ (mean age 67years). Skin samples measuring $1 \mathrm{~cm}(\mathrm{~L}) \times 0.5 \mathrm{~cm}(\mathrm{~B})$ were taken from the centre of the sole. Tissue was preserved in $10 \%$ formaldehyde for 48 hours and further histological techniques were followed.

Results: In females thickness of the epidermis (Edp) of were observed $(866.07 \pm 22.20 \mu m)$, the thickness of stratum corneum $(470.43 \pm 21.68 \mu \mathrm{m})$, layers of stratum spinosum and stratum granulosum were 3 , number of rete pegs $(11.41 \pm 1.01)$, depth of rete pegs $(62.89 \pm 5.96 \mu \mathrm{m})$, the thickness of the papillary dermis and reticular dermis were $(182.23 \pm 14.60 \mu \mathrm{m})$ and $(871.20 \pm 16.93 \mu \mathrm{m})$ respectively. Whereas In males thickness of the epidermis(Edp) were observed $(562.10 \pm 24.91 \mu \mathrm{m})$, the thickness of stratum corneum was $(484.36 \pm 49.21 \mu \mathrm{m})$, layers of stratum spinosum and stratum granulosum were 3 and 1 respectively, the number of rete pegs $10.81 \pm 1.92$, depth of rete pegs $(103.04 \pm 18.95 \mu \mathrm{m})$, thicknesses of the papillary dermis and reticular dermis was $(100.10 \pm 21.68 \mu \mathrm{m})$ and $(829.76 \pm 186.50 \mu \mathrm{m})$ respectively.

Conclusion: Females showed thicker epidermis as well as dermis in comparison to males. Females had more number rete pegs but these are shallower than males.

Key Words: Sole, Epidermis, Stratum corneum, Rete pegs, Thickness, Dermis

\section{INTRODUCTION}

Skin is one of the visible and largest organ of the body and unsheathes all other organ. ${ }^{1}$ It protects against various external physical, chemical, and biological assailants, as well as prevents excess water loss from body. ${ }^{2}$ Variations in skin reaction depend upon its epidermal and dermal thickness, and distribution of epidermal appendages. ${ }^{3}$ Accurate values of thickness of epidermis of the skin and its variation with body site, sex and age are important in the fields of medical and biological research.

A wide variety of agents encountered in the workplace may cause irritation, injury, sensitization, infection or discolouration in the skin of the exposed worker. Some agents even can induce cancerous changes in the skin. ${ }^{4}$ Variations in thickness of the skin can affect the absorption of ionizing radiations. This is particularly important in the nuclear power industry where the dose to the basal layer of skin (deepest epidermal layer) can set a limit on time, during which operators are allowed to perform work. Diffusion of a radioactively labelled compound occurs through the epidermis and dermis into the blood vascular system of living subjects. Hence, it is essential to have values of epidermal thickness so that measurements can be interpreted in terms of dose to the basal layer. Protective measures against exposure of radioactive components are dependent on the skin thickness of various exposed parts. In absence of quantitative data, some thick parts may be unnecessarily protected or vice versa. ${ }^{3}$

\section{Corresponding Author:}

Dande Kaweri, Post Graduate Scholar, Department of Anatomy, King George's Medical University, UP, Lucknow, India. Mobile: 9685682713; Email: kdande03@gmail.com

ISSN: $2231-2196$ (Print)

Received: 29.01 .2021
ISSN: 0975-5241 (Online)

Revised: 02.04 .2021
Accepted: 27.05 .2021 
Several studies have been performed regarding various parameters of normal skin, both from cadaveric skin and from living healthy volunteers. ${ }^{3,5,6,7}$ Data is also available stating the histological changes in the skin with advancing age $e^{8,9,10}$ but a clear lacuna is seen in the documentation of differences in the histological features of the skin of the sole of human male and female. Therefore, the present study was carried out to formulate a basic background of differences that can set a standard to compare pathological and chronological changes.

\section{MATERIAL AND METHODS}

The present observational study was done in the Department of Anatomy, King George Medical University, Lucknow from August 2017 to September 2018. Ethical clearance was obtained from the Institutional Ethics Committee with IEC approval no.763/Ethics/R.Cell-18. For the present study, the skin was procured from the sole of six freshly embalmed human cadavers. Out of these three were males and three were females. The age of the male and female cadavers ranged between 60 to 70years (mean age 67years). Skin samples measuring $1 \mathrm{~cm}(L) \times 0.5 \mathrm{~cm}(B)$ were taken from the centre of the sole. The tissue was preserved in $10 \%$ formaldehyde for 48 hours.

Fixed tissue specimens were dehydrated through increasing concentrations $(30 \%, 50 \%, 70 \%, 90 \%$ and absolute) of ethanol. After clearing the tissue in xylene, embedding was done in paraffin wax. $5 \mu \mathrm{m}$ thick sections were cut using a rotary microtome. The whole thickness of the tissue was sectioned. Three regions, each containing 3 sections were chosen at the interval of 20 sections. Thus, for each site of each cadaver 3 slides were prepared. Hence, 3 slides were containing a total of 9 sections of each tissue from each cadaver. Slides were stained by Haematoxylin [Harris's] and Eosin stain. Each stained section was observed for 3 different fields thus for each cadaver 27 observations were obtained.

Following 8 quantitative parameters were observed for each slide;

1. The thickness of epidermis at dermal papilla (Edp)

2. The thickness of the stratum corneum (Tsc)

3. Number of layers of stratum spinosum at dermal papilla (ss)

4. Number of layers of stratum granulosum at dermal papilla (sg)

5. Depth of rete pegs (Drp)

6. Number of rete pegs (rp)

7. The thickness of the papillary dermis (Tpd)

8. The thickness of the reticular dermis (Trd)

The thickness of the above-mentioned parameters was measured with the help of ERMA's micrometre. The value of each parameter in the micrometre was entered into a word excel sheet. For each parameter, 9 values were taken and the mean value was calculated. The values were represented in Number and Mean \pm SD. The mean value of each parameter was compared for gender-wise changes by using the student t-test and the Mann-Whitney test. p-value less than 0.01 was considered highly significant. The statistical analysis was done using SPSS (Statistical Package for Social Sciences) Version 21.0 statistical Analysis Software. The microphotography was done with the help of a device incorporated with software easy capture U S B 2.0 high-quality video and audio.

\section{RESULTS}

\section{Epidermis:}

The thickness of the epidermis at the dermal papilla of females was significantly higher as compared to males. The stratum corneum of the male sole was thicker as compared to females. The ratio of the thickness of epidermis at dermal papilla and stratum corneum was found to be higher among males as compared to females. Female skin showed more cell layers in stratum spinosum and stratum granulosum. These values were statistically significant also. In none of the specimens, stratum lucidum was observed. (Table 1, Fig $1.1,1,2)$

\section{Dermoepidermal Junction:}

The number of rete pegs was significantly higher in the female sole as compared to males. As far as depth of rete pegs was concerned, in the female sole, these were significantly much shallower than males. (Table1)

\section{Dermis:}

The thickness of the Papillary dermis, as well as the reticular dermis of female planter skin, is higher as compared to males. Statistically significant values were observed for the higher thickness of the papillary dermis of the female sole. (Table 1; Fig 1.1,1.2 )

\section{DISCUSSION}

The skin of the sole has the highest coefficient of friction as compared to the dorsum of the hand, anterior and posterior side of the forearm, middle anterior and posterior leg. ${ }^{11}$ It has been also shown that frictional properties can reflect the chemical and physical properties of the skin surface and thus depends on the physiological variations and pathological conditions of the skin. ${ }^{12}$

In the present study thickness of epidermis at dermal papilla, layers of stratum spinosum and stratum granulosum of the non-friction site of the sole was significantly higher in females. Thickness of stratum corneum, SC/Edp ratio ( propor- 
Table 1: Gender wise comparison of various skin parameters from sole Region

\begin{tabular}{|c|c|c|c|c|c|c|}
\hline & \multicolumn{2}{|c|}{ Female $(n=27)$} & \multicolumn{2}{|c|}{ Male $(n=27)$} & \multicolumn{2}{|c|}{ Statistical significance } \\
\hline & Mean & SD & Mean & SD & 't'/Z & 'p' \\
\hline Thickness of epidermis (Epi) & 866.07 & 22.20 & 562.10 & 24.91 & $47 \cdot 331$ & $<0.001$ \\
\hline Thickness of stratum corneum(sc) & 470.43 & 21.68 & $484 \cdot 36$ & 49.27 & -1.345 & 0.185 \\
\hline Edp:sc & 0.543 & 0.012 & 0.861 & 0.074 & -22.080 & $<0.001$ \\
\hline Layers of stratum spinoum(ss) & $3.48(3.00)$ & 0.51 & $2.44(3.00)$ & 0.64 & -5.035 & $<0.001$ \\
\hline Layers of stratum granulosum(sg)* & $3.00(3.00)$ & 0.88 & 1.67 & 0.48 & -5.134 & $<0.001$ \\
\hline Number of rete pegs & 11.41 & 1.01 & 10.81 & 1.92 & 1.418 & 0.162 \\
\hline Depth of rete pegs & 62.89 & $5 \cdot 96$ & 103.04 & 18.95 & -10.499 & $<0.001$ \\
\hline Thickness of papillary dermis & 182.23 & 14.60 & 100.10 & 21.68 & 16.330 & $<0.001$ \\
\hline Thickness of reticular dermis & 871.20 & 16.93 & 829.76 & 186.50 & 1.150 & 0.255 \\
\hline
\end{tabular}

tion of stratum corneum to whole thickness) was less than male. It can be appreciated that a higher number of layers or number of keratinocytes in stratum spinosum and granulosum resulted in greater thickness of epidermis in females. Also, in females greater number of rete pegs could have been responsible for contributing more basal keratinocytes and hence greater thickness of the epidermis. Relatively, the contribution of stratum corneum in total epidermal thickness was greater in males. In males, there was a greater depth of rete peg suggesting better interlocking to avoid greater frictional forces. In contrast, the thickness of both papillary, as well as reticular dermis, was more in females than males. So, the disparity in thickness of epidermis and dermis between genders suggest some regulation which is affected more than by mere its function (Table 1).

It is observed that collagen density and packing of fibrils in the dermis is influenced by age as well as sex. With increasing age, skin collagen decreases more rapidly than skin thickness so collagen density decreases. Thickness of the dermis is proportional to the collagen content and collagen density is less in females of all ages. Probably this could explain the reason for the greater thickness of female dermis than males in the present study.

Very little information on the microscopic topography of normal skin is available. Lee and Hwang reported the thickness of epidermis of palm and sole in the Korean population in the range of 601- $637 \mu \mathrm{m} .{ }^{13}$ Egawa $\mathrm{M}$ et al., found the thickness of stratum corneum of palm $173 \mu \mathrm{m}$ which is lesser than the thickness of stratum corneum of the palm of females but greater than males in the present study. ${ }^{14}$ These reports highlight the racial variations of skin thickness and suggest the need to create a reference model for quantitative characteristics of healthy skin from various sites in different populations.

Gender differences in the various parameters of skin could be attributed to estrogen. Recently estrogen receptors are demonstrated in the skin of mice. Shuster S et al., (1975) observed that the thickness of skin decreases linearly in males as early as, at the age of 20 in contrast it remains constant in females till 50 years and then it decreases. ${ }^{15}$ Waller JM et al., (2005) stated that the thickness of the epidermis decreases about $6.4 \%$ per decade on average whereas in postmenopausal women thickness decreases at a much higher rate than men. ${ }^{16}$ McCall ion Ret al., (1993) observed that the thickness of the dermis decreases up to $20 \%$ in both males and females. ${ }^{17}$ Whereas de Rigal J (1989) mentioned in his study that thickness of dermis of sun unexposed sites decreases after 80 years..$^{18}$

During weight-bearing activities, the feet are exposed to large forces, particularly in dynamic activity such as walking to normal rollover during the stance phase of gait. ${ }^{19}$ Suprapapillary thickness of the epidermis, layers of stratum spinosum and stratum granulosum of the non-friction area of sole were statistically. The pressure under the plantar surface during walking varies per foot area because of various factors related higher in females than in males (table1). Whereas the thickness of the stratum corneum was higher in males. The number of rete peg was more in females in contrast to the depth of rete pegs which was more in males and was statistically significant too (table 1). The thickness of papillary and reticular dermis was higher in females than males. Igarashi $\mathrm{T}$ et al., (2005) reported in their article the thickness of epidermis of sole $1.6 \mathrm{~mm}$ which was greater than the thickness of the epidermis $(0.714 \pm 0.155 \mathrm{~mm})$ at the dermal papilla of the present study. ${ }^{20}$

Even though subjects from both sexes belonged to the same age group, clear and statistically significant histological differences were observed in the skin of the sole. Females showed thicker epidermis and dermis in comparison to males. Females had more number rete pegs but these are shallower than males.

\section{CONCLUSION}

Females showed thicker epidermis as well as dermis in comparison to males. The higher number of layers or number 
of keratinocytes in stratum spinosum as well as in stratum granulosum resulted in greater thickness of epidermis in females. In females, the greater number of rete pegs as compared to males could have been responsible for contributing more basal keratinocytes and hence greater thickness of the epidermis.

\section{ACKNOWLEDGEMENT}

The authors acknowledge the head of the Department of Anatomy, King George Medical University, Lucknow and the immense help received from the scholars whose articles are cited and included in references of this manuscript. The authors are also grateful to authors/editors/publishers of all those articles, journals and books from where the literature for this article has been reviewed and discussed.

Conflict of interest: There is no conflict of interest.

\section{Source of funding: Nil.}

Author's contribution: All authors contribute to dissection, slide preparations and compiling this observational study.

\section{REFERENCES}

1. Venkataram M. Textbook on cutaneous and aesthetic sugery. Jaypee Bro Medical Publi pvt.Ltd.2012;1:4-5.

2. Kolarsick PAJ, Kolarsick MA, Goodwin C. Anatomy and physiology of skin: Erratum. J of the Dermatol Nurses Asso: 2011; 3( 6):366.

3. Whitton JT, Everall JD. The thickness of the epidermis. British $\mathrm{j}$ of Dermatol 1973;89: 467-476.

4. Occupational disease of the skin. Australian government publishing service. canberra.1990.3.

5. Holbrook KA, Odland GF. Regional differences in the thickness (cell layers) of the human stratum corneum: An ultrastructural analysis. J Invest Dermatol.1974; 62: 415-422.

6. Bergstresser PR, Taylor JR.Epidermal "turnover-time"- - a new examination.Br J Dermatol.1977;96: 503-506.
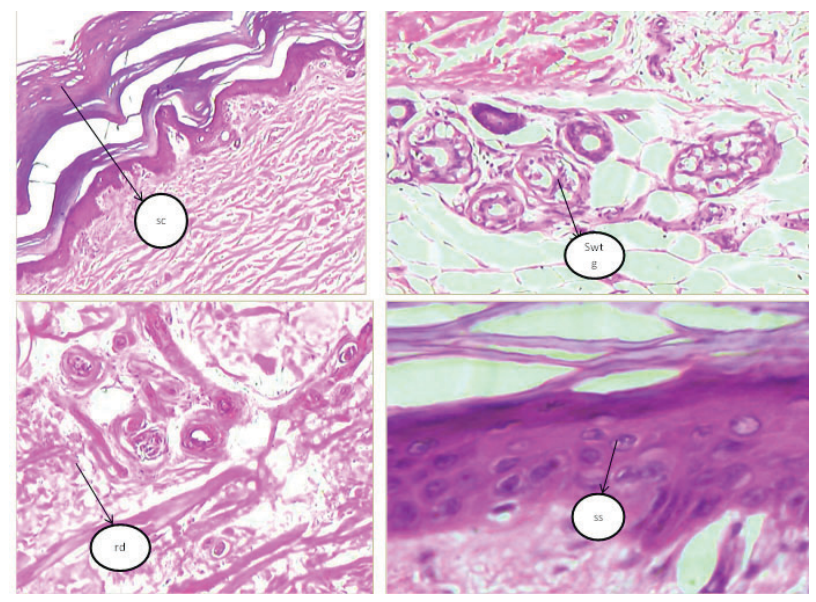

Figure 1.1: Stain: H\&E. Microphotograph of sole of male showing sc, swt g, rd \& ss in $4 \mathrm{X}, 10 \mathrm{X} \& 40 \mathrm{X}$ respectively.
7. Chopra K, Calva D, Sosin M, Tadisina KK, Banda BS, Cruz $\mathrm{CDL}$, et al. A comprehensive examination of the topographic thickness of the skin in the human face. Aesthetic Surgery J.2015; 35(8):1007-1013.

8. Habib MFA, Kadhim SS. Age-related Changes in Human Skin: Histological, Morphometric and Immunocytochemical Study Using S100.iraqi jms.2015;10(2):112-113.

9. Neerken S, Lucassen GW, Bisschop MA, Lenderink E. characterization of age-related effects in human skin: a comparative study that applies confocal laser scanning microscopy and optical coherence tomography. J of biomedical optics.2004;9(2):274-281.

10. Kawasaki K, Yamanishi K, Yamada H. Age-related morphometric changes of inner structures of the skin assessed by in vivo reflectance confocal microscopy. Int. J. Dermatol..2015;54:295-301.

11. Zhang M, Mak AFT. In vivo friction properties of skin. SAGE journal.1999;23(2):135-141.

12. Asserin J, Zohauani H, Philippe G H. Measurement of the friction coefficient of the human_skin_in_vivo_Quantification_of_ the_cutaneous_smoothness.Colloids and surfaces B: Biointerfaces .2000,19(1):1-12

13. Y Lee, K Hwang. Skin thickness of Korean adults. Surg and Radio Anatomy. 2002;24(3-4):183-189.

14. Egawa M, Hirao T, Takahashi M. In vivo Estimation of Stratum Corneum Thickness from Water Concentration Profiles Obtained with Raman Spectroscopy. Acta Dermatol Venereol 2007; 87: 4-8.

15. Shuster S, Black MM, Mcvitie E. The influence of age and sex on skin thickness, skin collagen and density. Br J Dermatol.1975;93:639-643.

16. Waller JM, Maibach HI. Age and skin structure and function, a qualitative approach(1): blood flow, $\mathrm{PH}$ thickness and ultrasound echogenicity. Skin res technol.2005;11:221-235.

17. Mccallion R, Wan A. Dry and photoaged skin: manifestations and management. J Clin Pharm Ther.1993;18:15-32.

18. Rigal J, Escoffier C, Querleux B, Faivre B, Agache P. Assessment of aging of the human skin by in vivo ultrasonic imaging. $j$ invest dermatol.1989;93:621-625.

19. Deursen V. Mechanical loading and off-loading of the plantar surface of the diabetic foot. Clin Infect Dis.2004;1(39):87-91.

20. Igarashi T, Nishino K, Nayar SK. The appearance of human skin. Foundations and Trends in Computer Graphics and Vision.2007;3(1): 4-5.
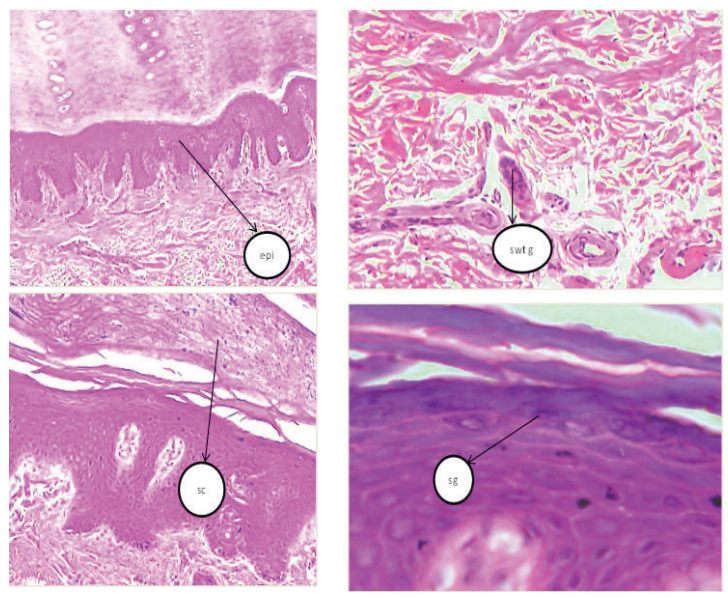

Figure 1.2: Stain: H\&E. Microphotograph of sole of female showing epi, swt g, sc \& sg in 4 X, 10X respectively. 